\title{
State and Rate Dependent Contact Line Dynamics over an Aging Soft Surface
}

\author{
Dongshi Guan $\odot,{ }^{1,2}$ Elisabeth Charlaix, ${ }^{3}$ and Penger Tong $\oplus^{2}$ \\ ${ }^{1}$ State Key Laboratory of Nonlinear Mechanics, Institute of Mechanics, Chinese Academy of Sciences, Beijing 100190, China \\ ${ }^{2}$ Department of Physics, Hong Kong University of Science and Technology, Clear Water Bay, Kowloon, Hong Kong \\ ${ }^{3}$ Laboratoire Interdisciplinaire de Physique, CNRS, Université Grenoble Alpes, Grenoble F-38000, France
}

(Received 21 October 2019; revised manuscript received 17 February 2020; accepted 22 April 2020; published 8 May 2020)

\begin{abstract}
We report direct atomic-force-microscope measurements of capillary force hysteresis $(\mathrm{CFH})$ of a circular contact line (CL) formed on a long glass fiber, which is coated with a thin layer of soft polymer film and intersects a water-air interface. The measured CFH shows a distinct overshoot for the depinning of a static CL, and the overshoot amplitude grows logarithmically with both the hold time $\tau$ and fiber speed $V$. A unified model based on the slow growth of a wetting ridge and force-assisted barrier crossing is developed to explain the observed time (or state) and speed (or rate) dependent CL depinning dynamics over an aging soft surface. The experimental findings have important implications to a common class of problems involving depinning dynamics in a defect or roughness landscape, such as friction of solid interfaces.
\end{abstract}

DOI: 10.1103/PhysRevLett.124.188003

Pinning-depinning (or stick-slip) is a common phenomenon both in nature and in many engineering applications. It is observed in a variety of out-of-equilibrium disordered systems as a yield response to an external forcing. Examples include the dynamics of magnetic domain walls $[1,2]$ and vortex lines [3]; the solid friction [4,5]; the plastic deformation of crystals and glassy materials [6,7]; and in geological flows, such as snow avalanches and earthquakes on faults $[8,9]$. The pinning-depinning dynamics often exhibit a state dependent increase of pinning strength owing to the contact aging. Recent atomic force microscopy (AFM) measurements [10-13] using a nanoscopically sharp AFM tip sliding against a crystal surface revealed that the frictional aging for a single asperity at the nanoscale may arise from the strengthening of interfacial chemical bonds. At the macroscopic level, however, other mechanisms may contribute to contact aging, such as slow creeping of asperities in the contact area [14]. Because of the complexity of the phenomena owing to the extreme conditions and complex material parameters involved, our fundamental understanding of the pinning-depinning dynamics is often limited by fewer direct measurement of individual slip events and empirical modeling and simulations $[9,15,16]$.

In this Letter, we report a systematic study of the state and rate dependent pinning-depinning dynamics in a unique model system, namely, the moving contact line (CL) between a liquid-air interface and a heterogeneous solid surface made of a soft polymer film. Because of the physical roughness and chemical inhomogeneity on the polymer surface, the CL undergoes a pinning-depinning transition under a constant pulling $[17,18]$. This $C L$ pinning causes the contact angle $\theta$ between the liquid and solid surfaces to exhibit some hysteresis with the advancing contact angle $\theta_{a}$ being larger than the receding contact angle $\theta_{r}$ [19-21]. In the experiment, we use a recently developed "long-needle" AFM [22-25] to directly measure the capillary force acting on a circular CL. As shown in Fig. 1, the long needle is made of a thin glass fiber coated by a layer of temperature-sensitive poly( $N$-isopropylacrylamide) (PNIPAM) of $\sim 60 \mathrm{~nm}$ in thickness (see Supplemental Material [26] for more details). The vertical PNIPAM-coated glass fiber penetrates a liquidair interface, at which a circular CL is formed on the fiber

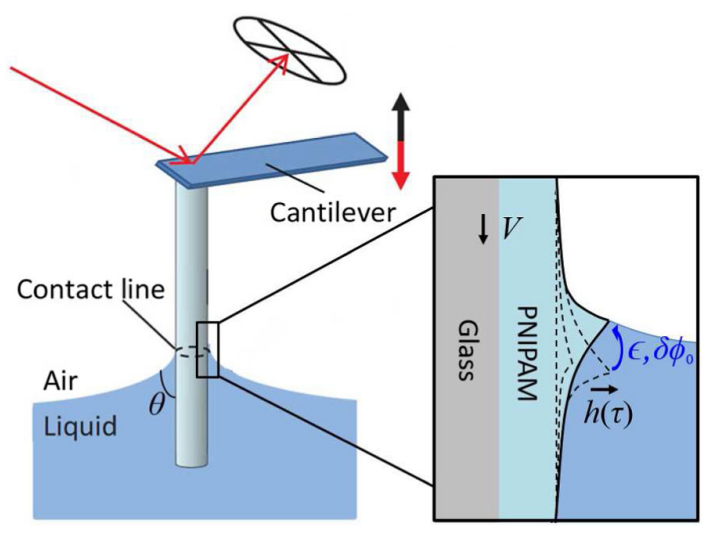

FIG. 1. Sketch of the AFM-based capillary force apparatus at a liquid-air interface. The "long needle" AFM involves a vertical glass fiber of diameter $d$ in the range $1-3 \mu \mathrm{m}$ and length 150-300 $\mu \mathrm{m}$, which is glued on to the front end of a rectangular cantilever beam. The surface of the glass fiber is coated with a thin layer of soft polymer film (PNIPAM). The inset indicates a slow growing wetting ridge (dashed lines) of height $h(\tau)$ formed beneath the three-phase CL. The solid line indicates the wetting ridge with a tangential displacement $\epsilon$ and corresponding tilted angle $\delta \phi_{0}$ resulting from the CL pulling when the fiber moves downward. 


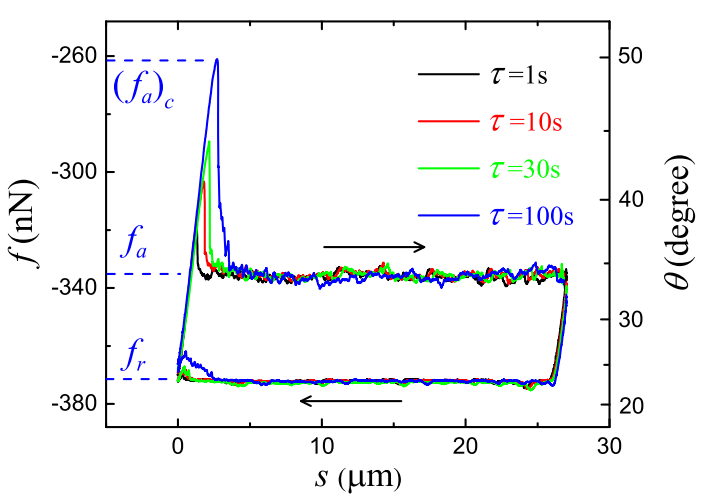

FIG. 2. Variations of the measured capillary force $f$ and corresponding contact angle $\theta$ [see Eq. (1)] when the glass fiber is first pushed downward (advancing $\rightarrow$ ) and then is pulled upward (receding $\leftarrow$ ) through a water-air interface. The measurements are made at a fixed fiber speed $V=10 \mu \mathrm{m} / \mathrm{s}$ and sample temperature $T=26^{\circ} \mathrm{C}$ for different hold times: $\tau=1$ (black), 10 (red), 30 (green), and $100 \mathrm{~s}$ (blue).

surface. The long-needle AFM can accurately measure the capillary force [22,31],

$$
f_{i}(s)=-\pi d \gamma \cos \theta_{i}(s),
$$

as a function of fiber traveling distance $s$ in the advancing (fiber moves downward, $i=a$ ) and receding (fiber moves upward, $i=r$ ) directions. Here, $\pi d$ is the length of CL on the fiber and $\gamma$ is the liquid-air interfacial tension. In this experiment, we use the fiber with diameter $d \simeq 1.8 \mu \mathrm{m}$. The sign of $f_{i}$ is defined as $f_{i} \leq 0$ for $\theta_{i} \leq 90^{\circ}$ and $f_{i}>0$ for $\theta_{i}>90^{\circ}$.

Figure 2 shows the typical CFH loops obtained when the PNIPAM-coated fiber is first pushed downward (advancing $\rightarrow)$ and then is pulled upward (receding $\leftarrow$ ) through a water-air interface at a constant speed $V$. Before the fiber starts to move downward, it was partially immersed in the water and was held stationary for a hold time $\tau$. When the fiber advances $(\rightarrow)$, the pinned interface is stretched, causing a sharp linear increase in $f$ with the distance traveled $s$, as shown by a straight line on the left side of the loop. When the restoring force of the liquid interface reaches its maximal value, $\left(f_{a}\right)_{c}$, the CL depins with an abrupt decrease in $f$ and then begins a steady stick-slip motion with the measured $f$ fluctuating around a mean value $f_{a}$ in the force curve. It is seen that the overshoot value, $\left(f_{a}\right)_{c}-f_{a}$, increases with the hold time $\tau$. When the direction of the fiber motion is reversed $(\leftarrow)$, the interface is pinned again and is stretched with the measured $f$ decreasing linearly with $s$ (right side of the loop). The CL then depins and the measured $f$ reaches a steady-state value $f_{r}$ in the receding direction. In this case, no overshoot is observed.

Figure 3 shows another set of CFH loops, which are obtained at different fiber speeds $V$ while the hold time $\tau$ is

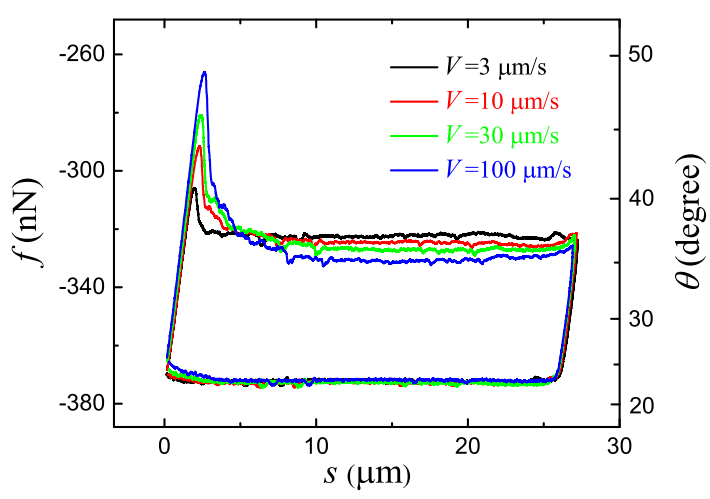

FIG. 3. Variations of the measured $f$ and corresponding $\theta$ as a function of traveling distance $s$ for different fiber speeds: $V=3$ (black), 10 (red), 30 (green), and $100 \mu \mathrm{m} / \mathrm{s}$ (blue). The measurements are made at a fixed hold time $\tau=1 \mathrm{~s}$ and sample temperature $T=32^{\circ} \mathrm{C}$.

kept at a fixed value. Each CFH loop consists of a sharp overshoot on the left side of the loop in the advancing direction, followed by a steady-state value of $f_{a}$. The overshoot increases with the fiber speed $V$, whereas the measured $f_{a}$ shows an opposite speed dependence, which decreases with increasing $V$. In the receding direction, neither the overshoot nor speed dependence is observed in the measured $f_{r}$.

Figures 2 and 3 reveal several interesting features of the CL dynamics over a soft surface. (i) The magnitude of the overshoot in the capillary force is found to depend both on the pinning state (or pinning age), i.e., the hold time $\tau$, and on the pulling rate, i.e., the fiber speed $V$. This state and rate dependent overshoot is not observed for a hard surface (such as a pure glass fiber) with various liquid-air interfaces [25]. (ii) The overshoot is strengthened with increasing $V$, whereas the steady-state value $f_{a}$ for an advancing CL shows an opposite $V$ dependence. This behavior suggests that the depinning of a static CL differs from the continuous stick-slip motion of a moving CL. (iii) The CFH loop is asymmetric with a large state and rate dependent overshoot only in the advancing direction. The advancing curve in the steady state also reveals relatively large fluctuations compared with those in the receding curve. In previous experiments [23-25], we found that the advancing CL is pinned primarily by the nonwetting (repulsive) defects, whereas the receding $\mathrm{CL}$ is pinned primarily by the wetting (attractive) defects. These two different types of defects were found to coexist on ambient solid surfaces [25]. For the PNIPAM-coated glass fiber, the polymer network behaves like nonwetting defects, so that the $\mathrm{CL}$ is pinned predominantly in the advancing direction. This explains why the overshoot and $V$ dependence occur only in the advancing direction but not in the receding direction.

To further quantify the overshoot in the advancing direction, we define the overshoot amplitude per unit length as $\Delta F_{\text {os }}(\tau, V) \equiv\left[\left(f_{a}\right)_{c}-f_{r}\right] /(\pi d)$, which is a 


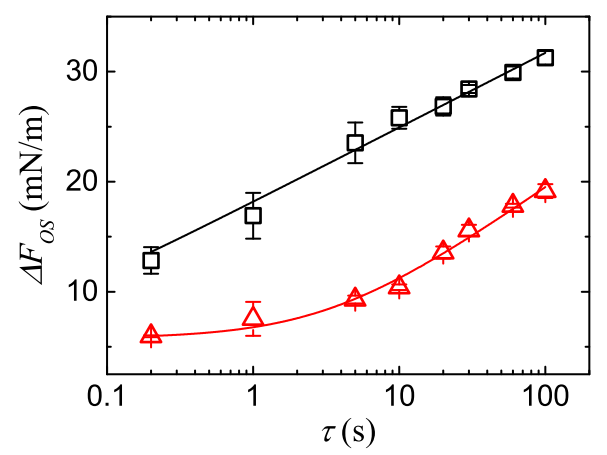

FIG. 4. Measured overshoot amplitude $\Delta F_{\text {os }}(\tau, V)$ as a function of hold time $\tau$ at a fixed fiber speed $V=10 \mu \mathrm{m} / \mathrm{s}$. The measurements are made at two sample temperatures: $T=$ $26^{\circ} \mathrm{C}$ (red triangles) and $T=32^{\circ} \mathrm{C}$ (black squares). The error bars show the standard deviation of the measurements. The solid lines are the fits of Eq. (2) to the data points with $A\left(26^{\circ} \mathrm{C}\right)=$ $3.99 \pm 0.25 \mathrm{mN} / \mathrm{m}, \tau_{0}\left(26^{\circ} \mathrm{C}\right)=3.4 \pm 0.5 \mathrm{~s}$, and $A\left(32^{\circ} \mathrm{C}\right)=$ $2.97 \pm 0.20 \mathrm{mN} / \mathrm{m}, \tau_{0}\left(32^{\circ} \mathrm{C}\right)=0.05 \pm 0.02 \mathrm{~s}$.

function of hold time $\tau$ and fiber speed $V$. Here $f_{r}$ is taken as a reference and it does not change with $\tau$ and $V$. Figure 4 shows how the measured $\Delta F_{\text {os }}(\tau, V)$ changes with $\tau$ at a fixed value of $V$. The measured $\Delta F_{\text {os }}(\tau, V)$ at a fixed $V$ shows an approximate $\ln (\tau)$ dependence for large values of $\tau$, which is a straight line in the linear-log plot. PNIPAM is a temperature-sensitive polymer with a lower critical solution temperature (LCST) around $32^{\circ}$, above which the polymer network undergoes a reversible phase transition from a swollen hydrated state to a shrunken dehydrated state, which is more hydrophobic and stiffer [32]. It is seen that the measured $\Delta F_{\text {os }}(\tau, V)$ for PNIPAM near its LCST is much larger than that at a lower temperature.

Figure 5 shows how the measured $\Delta F_{\text {os }}(\tau, V)$ (black symbols) changes with $V$ at a fixed value of $\tau$. The measured $\Delta F_{\text {os }}(\tau, V)$ at both temperatures shows a $\ln (V)$ dependence as well. For comparison, we also show, in Fig. 5, the measured $\Delta F_{a}(V)=\left(f_{a}-f_{r}\right) /(\pi d)$ (red symbols) for a moving CL at the steady state as a function of $V$. It is seen that while the measured $\Delta F_{a}(V)$ still exhibits a $\ln (V)$ dependence at both temperatures, it shows a different trend that decreases with increasing $V$. Note that $\Delta F_{a}(V)$ is independent of $\tau$, as shown in Fig. 2.

Similar logarithmic time dependence was also observed for the static fiction between two solid surfaces made of rocks, polymers, and other materials [12,13,33-35]. It was shown recently $[12,13]$ that the frictional aging for nanoscale silica contacts is caused by the progressive formation of interfacial chemical bonds under a normal compression. In our case, however, the logarithmic growth of the overshoot is caused by the formation of a "wetting ridge" below the CL, which is developed under the constant pulling of the normal component of the capillary force acting on the soft PNIPAM surface [26,36]. This wetting ridge has been observed on surfaces made of various soft materials, such as silicone rubber [37], silicone gel [38,39], and elastomers

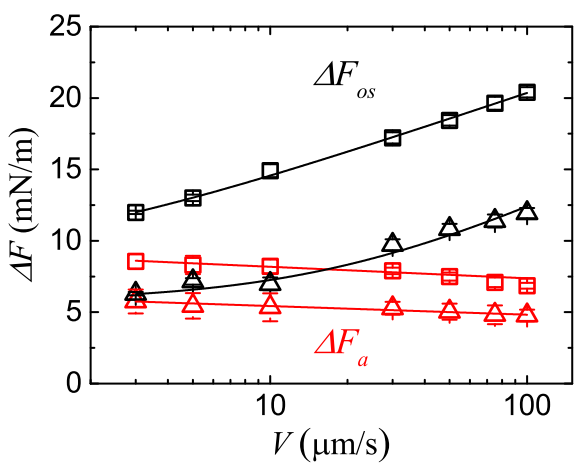

FIG. 5. Comparison between the measured $\Delta F_{\text {os }}(\tau, V)$ for a static CL (black symbols) and $\Delta F_{a}(V)$ for a moving CL (red symbols) both as a function of fiber speed $V$. The measurements are made at a fixed hold time $\tau=1 \mathrm{~s}$ and at temperatures $T=26{ }^{\circ} \mathrm{C}$ (triangles) and $32{ }^{\circ} \mathrm{C}$ (squares). The error bars show the standard deviation of the measurements. The black lines are the fits of Eq. (2) to the data points with $B\left(26^{\circ} \mathrm{C}\right)=3.71 \pm 0.50 \mathrm{mN} / \mathrm{m}, \quad V_{0}\left(26^{\circ} \mathrm{C}\right)=19.6 \pm 5.0 \mu \mathrm{m} / \mathrm{s}$ and $B\left(32^{\circ} \mathrm{C}\right)=2.63 \pm 0.40 \mathrm{mN} / \mathrm{m}, \quad V_{0}\left(32^{\circ} \mathrm{C}\right)=1.25 \pm$ $0.40 \mu \mathrm{m} / \mathrm{s}$. The red lines are fits of Eq. (3) to the data points with $(B-A)\left(26^{\circ} \mathrm{C}\right)=-0.26 \pm 0.02 \mathrm{mN} / \mathrm{m}$ and $(B-A)\left(32^{\circ} \mathrm{C}\right)=$ $-0.35 \pm 0.02 \mathrm{mN} / \mathrm{m}$.

$[40,41]$. For all these materials, the wetting ridge was found to act as a physical barrier that hinders the CL motion. It was shown [41] that the ridge height grows logarithmically in time, i.e., $\delta h(\tau) \sim h_{0} \ln \left(1+\tau / \tau_{0}\right)$, where $\delta h(\tau)$ is the height variation of the ridge and $h_{0}$ is a characteristic length of the system (for a thin film, $h_{0}$ is the film thickness [40]). The growth rate, $\tau_{0}^{-1}=D / h_{0}^{2}$, of the wetting ridge is limited by the diffusion of the solvent through the PNIPAM network with $D$ being the diffusivity of the solvent in the poroelastic material.

When the fiber advances, the CL is pulled by the restoring force of the liquid interface so that the ridge is pulled away from its static position by an amount $\epsilon$, as illustrated in the inset of Fig. 1. The displacement $\epsilon$ can be written as $\epsilon(\tau)=h(\tau) \tan \left(\delta \phi_{0}\right) \simeq h(\tau) \delta \phi_{0}$, where $\delta \phi_{0}$ is a typical (small) value of the tilted angle of the ridge under the tangential pulling. This elastic deformation of the ridge generates a dissipative straining force, $F_{S}(\tau) \simeq \pi d E \epsilon(\tau) \simeq$ $\pi d E h(\tau) \delta \phi_{0}$ [42], which deforms the defect landscape that the $\mathrm{CL}$ is anchored to [40]. As a result, the pinning barrier is strengthened in time, giving rise to the aging effect and an extra time-dependent force $F_{S}(\tau)$ needed to depin the CL [26]. In this way, the restored elastic energy in the deformed ridge is dissipated by the local rearrangement of the polymer network.

Even for a nondeforming substrate, one still needs a rupture force, $F_{R} \simeq \pi d(a / \lambda) f_{c}$, to break the pinning bonds between the CL and defects on the substrate so that the CL can move [23,24]. Here $a / \lambda$ is the defect line density with $\lambda$ being the defect size and $a \leq 1$ being a numerical constant, and $f_{c} \simeq 3 E_{b} / \lambda$ is the critical force needed to break a single defect bond with an energy barrier $E_{b}[43,44]$. When the 
fiber surface on which the CL is pinned moves at a constant speed $V$, the liquid interface is continuously stretched and exerts a time-dependent pulling force, $f_{s} \simeq k_{s} V t$, on the CL, where $k_{s} \sim \gamma$ is the spring constant of the liquid interface [25]. If the normalized loading rate $k_{s} V / f_{c}$ is smaller than the thermal activation rate $K_{0} \exp \left(-E_{b} / k_{B} T\right)$ (i.e., when the energy barrier $E_{b}$ is not too large compared with the thermal energy $k_{B} T$ ), thermal fluctuations can help the barrier crossing and the force needed to depin a single defect is less than $f_{c}$. In this case, one can show $[23,24,45,46]$ that $F_{R}(V) \simeq \pi d(a / \lambda)\left(2 k_{B} T / \lambda\right) \ln \left(1+V / V_{0}\right)$, where $V_{0}=$ $2 k_{B} T K_{0} \exp \left(-E_{b} / k_{B} T\right) /\left(e^{-\alpha} k_{s} \lambda\right)$ is a characteristic speed, $K_{0}$ is the attempt frequency, and $\alpha \simeq 0.577$ is the Euler constant [26].

By taking both the aging effect of the wetting ridge and thermal activation effect of the force-assisted barrier crossing into account, we obtain the CL depinning force per unit length, $\Delta F(\tau, V) \simeq\left[F_{S}(\tau)+F_{R}(V)\right] /(\pi d)$, as,

$$
\Delta F(\tau, V)=\Delta F_{0}+A \ln \left(1+\frac{\tau}{\tau_{0}}\right)+B \ln \left(1+\frac{V}{V_{0}}\right),
$$

where $\Delta F_{0}$ is the residual $\mathrm{CFH}$, which is independent of $\tau$ and $V$. The second and third terms on the right-hand side of Eq. (2) are, respectively, the state and rate dependent parts of the CFH with $A=E h_{0} \delta \phi_{0}$ and $B=(a / \lambda)\left(2 k_{B} T / \lambda\right)$. A similar equation was also obtained for the state and rate dependent friction of solid interfaces [34,35,47]. For a fixed value of $V, \Delta F(\tau, V)$ in Eq. (2) shows a $\ln \left(\tau / \tau_{0}\right)$ dependence for large values of $\tau / \tau_{0}$. The solid lines in Fig. 4 show the fits of Eq. (2) to the data with $A$ and $\tau_{0}$ as two fitting parameters. In the fitting, the value of the constant, $\Delta F_{0}+$ $B \ln \left(1+V / V_{0}\right) \quad\left(\simeq \Delta F_{a}\right)$, has been predetermined in the experiment at $\tau \simeq 0$ without the overshoot [26]. It is seen that Eq. (2) fits the data well.

Similarly, for a fixed value of $\tau, \Delta F(\tau, V)$ in Eq. (2) exhibits a $\ln \left(V / V_{0}\right)$ dependence for large values of $V / V_{0}$. The black lines in Fig. 5 show the fits of Eq. (2) to the data with $B$ and $V_{0}$ as two fitting parameters. In the fitting, the constant $\Delta F_{0}+A \ln \left(1+\tau / \tau_{0}\right)$ has been predetermined from the measured force relaxation when the advancing fiber suddenly stops with $V \simeq 0$ [26]. From the fitted values of $B$ and $V_{0}$, we obtain the energy barrier height $E_{b} \simeq$ $(10-15) k_{B} T$ and defect size $\lambda \simeq(1.2-1.4) \mathrm{nm}$ [26]. Similar values of $E_{b}$ and $\lambda$ were also obtained for the depinning of a static CL over a hard hydrophobic fiber surface without any aging effect [23,24].

Compared with the measured $\Delta F_{\text {os }}(\tau, V)$, which is an increasing function of $V$ for a static CL, the measured $\Delta F_{a}(V)$ for a moving CL exhibits a different trend that decreases with $V$, as shown in Fig. 5 (red symbols). For an advancing $\mathrm{CL}$ at a constant speed $V$, its contact with the underlying surface defects is continuously refreshed once the CL moves over a "memory" distance $\ell_{c}$. Therefore, the effective hold time for a moving CL is $\tau=\ell_{c} / V$. By substituting this definition of $\tau$ into Eq. (2) and taking the limit $\left(\ell_{c} / V\right) / \tau_{0} \gg 1$, we have

$\Delta F_{a}(V) \simeq \Delta F_{0}+(B-A) \ln \left(\frac{V}{V_{0}}\right)+A \ln \left(\frac{\ell_{c}}{V_{0} \tau_{0}}\right)$.

The red lines in Fig. 5 show the fits of Eq. (3) to the data with $B-A$ (slope) and an intercept as two fitting parameters. The fitted values of $B-A$ agree with the values of $A$ and $B$ obtained separately from the measured $\Delta F_{\text {os }}(\tau, V)$. Our finding that $A>B$ suggests the aging effect is more important for the soft PNIPAM surface.

The above results thus demonstrate that the aging effect of the wetting ridge causes the depinning force to exhibit a logarithmic growth in time for a static CL and speed weakening for a moving CL. We expect that these features are also applicable to other soft viscoelastic surfaces, as the formation of a wetting ridge introduces an extra viscoelastic dissipation for all soft materials. Furthermore, Eqs. (2) and (3) provide predictions about how the depinning force depends on the material parameters. First, the correlation distance $\ell_{c}$ can be obtained by extrapolating the two solid lines of $\Delta F_{\text {os }}$ and $\Delta F_{a}$ in Fig. 5 to the crossover point, $V \simeq 0.5 \mu \mathrm{m} / \mathrm{s}$ (at $32^{\circ} \mathrm{C}$ ), at which $\Delta F_{\text {os }}(\tau, V)=\Delta F_{a}(V)$ and thus $\tau=\ell_{c} / V$. With $\tau=1 \mathrm{~s}$, we find $\ell_{c} \simeq 0.5 \mu \mathrm{m}$. This value of $\ell_{c}$ is much larger than the defect size $\lambda$ $(\simeq 1.3 \mathrm{~nm}$ ), but is comparable to the typical slip distance, as shown in the measured capillary force fluctuations (see the magnified plot in Fig. S4 [26]).

Second, the aging effect depends sensitively on the poroelastic properties of the soft surface, which determine the growth rate of the wetting ridge $[41,48]$. With the temperature-sensitive PNIPAM, one can vary the poroelastic properties of the solid surface in situ by changing the sample temperature $T$. For example, at $T=32{ }^{\circ} \mathrm{C}$, the PNIPAM network starts to shrink and its surface becomes stiffer and more hydrophobic [26]. As a result, both the film thickness and pore size are reduced and so does the aging time $\tau_{0}$, as shown by the fittings in Fig. 4. Our experimental findings thus demonstrate a unique microscopic mechanism underpinning the state and rate dependent pinning-depinning dynamics, which has many important applications in microfluidics and nanotechnology. For example, by adjusting the substrate softness, one is able to modify the CL dynamics and further control the droplet evaporation and heat transfer $[49,50]$.

The authors wish to thank Dr. L. Bureau and Dr. B. Cross for useful discussions. This work was supported in part by NSFC under Grant No. 11972351 and the Pioneer Hundred Talents Program of CAS (D. G.) and by the Hong Kong RGC under Grants No. 16306418 (D. G. and P. T.), No. 16302718 (P. T.), and No. AoE/P-02/12 (P. T.). 
[1] G. Bertotti, Hysteresis in Magnetism: For Physicists, Material Scientists, and Engineers (Academic Press, San Diego, 1998).

[2] S. Zapperi, P. Cizeau, G. Durin, and H. E. Stanley, Phys. Rev. B 58, 6353 (1998).

[3] G. Blatter, M. V. Feigelman, V. B. Geshkenbein, A. I. Larkin, and V. M. Vinokur, Rev. Mod. Phys. 66, 1125 (1994).

[4] Y. Mo, K. T. Turner, and I. Szlufarska, Nature (London) 457, 1116 (2009).

[5] M. H. Müser, Phys. Rev. Lett. 100, 055504 (2008).

[6] D. M. Dimiduk, C. Woodward, R. LeSar, and M. D. Uchic, Science 312, 1188 (2006).

[7] M.-C. Miguel, A. Vespignani, S. Zapperi, J. Weiss, and J.-R. Grasso, Nature (London) 410, 667 (2001).

[8] J. M. Carson, J. S. Langer, and B. E. Shaw, Rev. Mod. Phys. 66, 657 (1994).

[9] H. Kawamura, T. Hatano, N. Kato, S. Biswas, and B. K. Chakrabarti, Rev. Mod. Phys. 84, 839 (2012).

[10] R. Erlandsson, G. Hadziioannou, C. M. Mate, G. M. McClelland, and S. Chiang, J. Chem. Phys. 89, 5190 (1988).

[11] Q. Li, Y. Dong, D. Perez, A. Martini, and R. W. Carpick, Phys. Rev. Lett. 106, 126101 (2011).

[12] Q. Li, T. E. Tullis, D. Goldsby, and R. W. Carpick, Nature (London) 480, 233 (2011).

[13] K. Tian, N. N. Gosvami, D. L. Goldsby, Y. Liu, I. Szlufarska, and R. W. Carpick, Phys. Rev. Lett. 118, 076103 (2017).

[14] J. H. Dieterich and B. D. Kilgore, Pure Appl. Geophys. 143, 283 (1994).

[15] S. Santucci, K. T. Tallakstad, L. Angheluta, L. Laurson, R. Toussaint, and K. J. Maloy, Phil. Trans. R. Soc. A 377, 20170394 (2018).

[16] L.-H. Tang, in Collective Transport and Depinning in Encyclopedia of Complexity and Systems Science, edited by R. A. Meyers (Springer, New York, 2009).

[17] P.-G. de Gennes, Rev. Mod. Phys. 57, 827 (1985).

[18] S. Varagnolo, D. Ferraro, P. Fantinel, M. Pierno, G. Mistura, G. Amati, L. Biferale, and M. Sbragaglia, Phys. Rev. Lett. 111, 066101 (2013).

[19] L. Leger and J.-F. Joanny, Rep. Prog. Phys. 55, 431 (1992).

[20] E. L. Decker and S. Garoff, J. Adhes. 63, 159 (1997).

[21] D. Bonn, J. Eggers, J. Indekeu, J. Meunier, and E. Rolley, Rev. Mod. Phys. 81, 739 (2009).

[22] X.-M. Xiong, S. Guo, Z.-L. Xu, P. Sheng, and P. Tong, Phys. Rev. E 80, 061604 (2009).

[23] D. Guan, Y. J. Wang, E. Charlaix, and P. Tong, Phys. Rev. Lett. 116, 066102 (2016).
[24] D. Guan, Y. J. Wang, E. Charlaix, and P. Tong, Phys. Rev. E. 94, 042802 (2016).

[25] Y. J. Wang, S. Guo, H.-Y. Chen, and P. Tong, Phys. Rev. E 93, 052802 (2016).

[26] See Supplemental Material at http://link.aps.org/ supplemental/10.1103/PhysRevLett.124.188003 for more details about sample preparation, theoretical model, and data analysis, which includes Refs. [27-30].

[27] R. W. Friddle, Phys. Rev. Lett. 100, 138302 (2008).

[28] G. I. Bell, Science 200, 618 (1978).

[29] T. Baumberger, P. Berthoud, and C. Caroli, Phys. Rev. B 60, 3928 (1999).

[30] S. Guo, M. Gao, X. Xiong, Y. J. Wang, X. Wang, P. Sheng, and P. Tong, Phys. Rev. Lett. 111, 026101 (2013).

[31] J.-M. Di Meglio and D. Quéré, Europhys. Lett. 11, 163 (1990).

[32] T. R. Matzelle, G. Geuskens, and N. Kruse, Macromolecules 36, 2926 (2003).

[33] J. H. Dieterich, J. Geophys. Res. 77, 3690 (1972).

[34] P. Berthoud, T. Baumberger, C. G'Sell, and J.-M. Hiver, Phys. Rev. B 59, 14313 (1999).

[35] C. Marone, Annu. Rev. Earth Planet Sci. 26, 643 (1998).

[36] R. W. Style, A. Jagota, C.-Y. Hui, and E. R. Dufresne, Annu. Rev. Condens. Matter Phys. 8, 99 (2017).

[37] A. Carré, J.-C. Gastel, and M. E. R. Shanahan, Nature (London) 379, 432 (1996).

[38] S. J. Park, B. M. Weon, J. S. Lee, J. Lee, J. Kim, and J. H. Je, Nat. Commun. 5, 4369 (2014).

[39] S. Karpitschka, S. Das, M. van Gorcum, H. Perrin, B. Andreotti, and J. H. Snoeijer, Nat. Commun. 6, 7891 (2015).

[40] R. Lhermerout, H. Perrin, E. Rolley, B. Andreotti, and K. Davitt, Nat. Commun. 7, 12545 (2016).

[41] M. Zhao, F. Lequeux, T. Narita, M. Roché, L. Limat, and J. Dervaux, Soft Matter 14, 61 (2018).

[42] J. E. Sader, J. Appl. Phys. 84, 64 (1998).

[43] A. Garg, Phys. Rev. B 51, 15592 (1995).

[44] X.-G. Ma, P.-Y. Lai, B. J. Ackerson, and P. Tong, Soft Matter 11, 1182 (2015).

[45] H. J. Lin, H. Y. Chen, Y. J. Sheng, and H. K. Tsao, Phys. Rev. Lett. 98, 088304 (2007).

[46] H. Perrin, R. Lhermerout, K. Davitt, E. Rolley, and B. Andreotti, Phys. Rev. Lett. 116, 184502 (2016).

[47] H. Kawamura, T. Hatano, N. Kato, S. Biswas, and B. K. Chakrabarti, Rev. Mod. Phys. 84, 839 (2012).

[48] J. Yoon, S. Cai, Z. Suo, and R. C. Hayward, Soft Matter 6, 6004 (2010).

[49] M. C. Lopes and E. Bonaccurso, Soft Matter 8, 7875 (2012).

[50] E. Y. Gatapova, A. M. Shonina, A. I. Safonov, V. S. Sulyaeva, and O. A. Kabov, Soft Matter 14, 1811 (2018). 